\section{Conventional echo color Doppler versus ULA-OP in the assessment of venous flow model}

\author{
Valentina Tavoni, ${ }^{1,2}$ Francesco Sisini, ${ }^{1,2}$ \\ Giovanni Di Domenico, ${ }^{1}$ \\ Nadiya Mohammed, ${ }^{1}$ Giacomo Gadda, ${ }^{1}$ \\ Francesca Calderoni, ${ }^{1}$ \\ Mauro Gambaccini ${ }^{1}$ \\ ${ }^{1}$ Department of Physics and Earth \\ Sciences; ${ }^{2}$ Vascular Diseases Center, \\ University of Ferrara, Italy
}

\begin{abstract}
The quantification of venous flows can be obtained by multiplying cross sectional area, measured on a B-mode video-clip, by velocity values, obtained with Doppler measurements. The Doppler angle between ultrasound (US) line and blood flow requires a manual adjustment. Establishing this parameter is critical in order to calculate blood velocity. However, the operator dependency gives high variable results. It is worth noting that a new class of vector Doppler devices can enhance the accuracy and precision of measurements. Such technology uses a double US line that leads to automatically know the Doppler angle. By comparing in an in vitro model of venous flow conventional echo color Doppler (ECD) equipment with the new device, we found a better minimal difference between the latter and the nominal flow rate $(20 \%)$. On the contrary, the comparison with conventional ECD showed a difference ranging between $2 \%$ and $43 \%$, according to the possible settings of the equipment. Our study demonstrates a better accuracy of the experimental device with respect to conventional $\mathrm{ECD}$ in measuring the venous flow rate.
\end{abstract}

\section{Introduction}

Ultrasound (US) devices are becoming very attractive in the clinical environment, particularly for medical diagnostics. This technology is used also in the cardiovascular field to quantify blood flows. ${ }^{1,2}$ The flow is calculated by multiplying blood velocity in a vessel, obtained with Doppler measurements, by its cross sectional area (CSA), measured on a B-mode video-clip. ${ }^{3-6}$

Differently from other methods used for the same purpose (for example phlebography or functional magnetic resonance imaging), such technology allows to do non- invasive, cheap and safe examinations. Several studies published in the last decades highlighted a good reproducibility among US technicians trained in investigation of cerebral venous return. ${ }^{7-9}$

However, highly variable results still exist because Doppler measurements of blood velocity are strongly affected by two types of error, which are systematic and random. ${ }^{1}$ A systematic error depends on the instrumentation: in order to overcome such problem, a calibration of the devices is necessary. This practice allows to know any device limits and to evaluate the correction factor to apply on each measurement.

Since the US technology is operator dependant, a random error is related to the operating mode of the specialist who is doing the US examination. In order to reduce this dependency, several investigators attempted to measure the cerebral venous outflow by means of quantitative US protocol. Nevertheless, such a protocol required very skilled personnel and a long period of time to collect measurements. , $^{2,10,11}$

In particular, the technician has to pay attention when positioning the US probe along the vessels. He shall avoid pressing on the subject's skin, because the veins could collapse and change their CSA. Moreover, he has to manually adjust the orientation of the US cursor, which must be parallel to the blood flow. ${ }^{1}$ Such operation allows the system to calculate the insonation angle between the US line and the blood flow. The choice of this parameter is fundamental, because the Doppler equation, required to determine blood velocity, depends on the inverse of the cosine of the angle.

Furthermore, it is known that veins tend to be tortuous, making measurements a challenge. In some cases, the presence of valves within the vessels also may lead to turbulences in blood flow. For example, the internal jugular vein region right before the subclavian junction is an anatomical point exposed to flow turbulence, both because of the vessel direction and since it is the most prevalent valvular location. In this venous segment, an error of about $30 \%$ in measuring the flow rate has been estimated, which is due to the uncertain velocity assessment. ${ }^{2,12}$

Another source of variability during US examination is the intrinsic spectral broadening (ISB). ISB is a broadening of the Doppler spectrum due to the mechanism of delivering and receiving acoustic energy. ${ }^{13}$

To overcome some of these sources of errors a new experimental US platform was carried out. ${ }^{14,15}$ Such device uses a double US line, that allows to measure blood velocity by calculating the Doppler angle of
Correspondence: Valentina Tavoni, Department of Physics and Earth Sciences, University of Ferrara, via Mondaini 7, 42122 Reggio Emilia, Italy.

E-mail: valentina.tavoni@unife.it

Key words: Diagnosis with ultrasound; echo color Doppler; ULA-OP; venous blood flow; angle of insonation.

Acknowledgements: the authors would like to thank Alessandro Ramalli of the Microelectronic Systems Design laboratory of the University of Florence for helping during the measurements with ULA-OP.

Funding: this research was supported by grant of the Ministry of Health RF-2013-02358029, by the association CCSVI nella Sclerosi Multipla and by the University of Ferrara.

Contributions: VT, FS, FC, GG and MG designed and implemented the experiments; VT, FC and GG collected the data; VT, FS and GDD analysed data and interpreted the results; VT and NM wrote the main manuscript; all authors reviewed the manuscript.

Conflict of interest: the authors declare no potential conflict of interest

Award: this work was awarded with the poster first place to the $7^{\text {th }}$ International Society of Neurovascular Disease Meeting, in Taormina, Sicily, Italy

Received for publication: 9 June 2017 Revision received: 2 August 2017. Accepted for publication: 2 August 2017.

This work is licensed under a Creative Commons Attribution 4.0 License (by-nc 4.0).

(C) Copyright V. Tavoni et al., 2017

Licensee PAGEPress, Italy

Veins and Lymphatics 2017; 6:6841

doi:10.4081/vl.2017.6841

insonation in an instantaneous and automatic way. Therefore, this technology has a nominal better accuracy and precision than standard devices.

The aim of this paper is to compare the accuracy and precision of conventional echo color Doppler (ECD) equipment with those of the new one, in a model of venous flow.

\section{Materials and Methods}

The comparison of scanners flow estimations was performed through an in vitro study with a closed circuit, composed by a 
homemade neck phantom and a gear pump that generates a static volume flow (Figure 1). The neck phantom was created with a plastic tube, with circular CSA equal to $(0.5 \pm 0.1) \mathrm{cm}^{2}$, inserted into a PMMA box $(20.0 \times 12.0 \times 20.5 \mathrm{~cm})$ full of water. The tube was fixed to a goniometer indicating the insonation angle, i.e. the angle between the US line and the flow direction. The pump was used to drive blood mimicking fluid (BMF) (CIRS, Model 046, Norfolk, Virginia, USA) simulating venous blood flow. The BMF is a liquid with the same acoustic properties of the blood. The tank containing the BMF and a turbine flowmeter were also inserted in the circuit (Figure 1). The flowmeter, powered by a $9 \mathrm{~V}$ battery and connected to an oscilloscope, was used to calibrate the pump.

The test was done using two devices: equipment A (Conventional ECD device) and equipment B (ULA-OP platform, Florence, Italy) connected to a PC with ULA-OP starter software. The US probe used during the tests is a linear array probe (LA523, Esaote S.P.A., Florence, Italy). This was fastened over the circuit to a manual positioner, which allows moving the probe in very small steps and fixing it in the desired position.

During each test the flow set in the pump was varied between $(150 \pm 1) \mathrm{mL} / \mathrm{min}$ and $(650 \pm 1) \mathrm{mL} / \mathrm{min}$ in steps of $(100 \pm 1)$ $\mathrm{mL} / \mathrm{min}$.

The repeatability was tested by reiterating the same measurements during one experimental session, for five times with the equipment $\mathrm{A}$ and for twenty times with the equipment B. Moreover, the reproducibility was measured by repeating the entire procedure in different experimental sessions in order to have some statistics, for five times with the equipment $\mathrm{A}$ and for twenty times with the equipment $\mathrm{B}$.

\section{Calibration test}

A preliminary calibration test was taken: this procedure consists in comparing the flow values set in the pump and the values measured by the flowmeter. The flowmeter gives a pulsed electric signal for each cycle, whose frequency was measured with an oscilloscope. The FFT mode of the oscilloscope provides us with the main frequency $f$, which is proportional to the measured flow $\Phi_{\text {flowmeter, as described by the }}$ equation:

$\Phi_{\text {flowmeter }}=\mathrm{f} / \mathrm{k}$

with $\mathrm{k}=22,000$ pulse/L.

The flow values measured with the flowmeter and those set in the pump were verified to be not significantly different by a paired t-test. Therefore, the nominal values set was constructed with flow values set in the pump $\left(\Phi_{\text {pump }}\right)$. These were converted into velocity values through the CSA of the tube since $\mathrm{v}=\Phi_{\text {pump }} / \mathrm{CSA}$.

\section{Test with equipment $A$}

The measurements were performed by positioning the insonation angle at $30^{\circ}, 45^{\circ}$ and $60^{\circ} \pm 1^{\circ}$. The choice of these angles follows the guidelines to obtain reliable measurements. ${ }^{16}$

For each flow value set in the pump, the Doppler velocity was measured inside the vessel by choosing two different sizes of sample volume (SV): the smallest $\mathrm{SV}$, i.e. 1 $\mathrm{mm}$, and the biggest $\mathrm{SV}$, which extends to include the whole diameter of the vessel, i.e. $8 \mathrm{~mm}$.

Since the flow is assumed to be laminar, both the mean time average velocity (TAV) and half of the peak TAV were compared with the velocity set in the pump.

The other parameters set in the system are reported in Table 1.

\section{Test with equipment $B$}

The US mode used to do the experiment was the B-MSD-SpTR-Mode. This mode allows to automatically adjusting the angle of insonation using a double US line. Moreover, this mode enables to calculate the peak velocity in the point within the vessel, where a marker for the focus of the measurement is located.

The experiment was performed by positioning the tube at an insonation angle equal to $80^{\circ} \pm 1^{\circ}$. The choice of this angle is given by the best setting suggested by the manufacturer to obtain reliable measurements (personal communication).

The system automatically displays only the measurement of the peak velocity.

The other parameters set in the system are reported in Table 2.

\section{Analysis of results}

For every different experimental setup, a set of measurements was collected. A set is composed by five or twenty velocity measurements corresponding to the single flow values set in the pump each time.

For every flow value of each set, mean values, standard deviations (SD) and coefficient of variation (COV) were calculated. Moreover, a simple linear regression was obtained for each set. Finally, paired t-test was used to state the null hypothesis that two set of velocity measurements were not significantly different from each other. All the tests done are reported in Table 3.

The tests with the equipment $\mathrm{A}$ were conducted with two different combination of insonation angle, SV and TAV: the suggested setup and the best setup. The first one is the setting suggested by the guidelines: ${ }^{16}$ insonation angle equal to $60^{\circ}, \mathrm{SV} 8$ $\mathrm{mm}$ and mean TAV. The second one instead is the setting that reproduces the closest measurements to the nominal values. The insonation angle for this setting is equal to $30^{\circ}$, SV $8 \mathrm{~mm}$ and peak TAV.

\section{Results}

From the verification of reproducibility and repeatability of measurements, a different COV was obtained for every flow values. The range of variation of $\mathrm{COV}$ expressed in percentage is reported in Table 4. The results of the t-test between nominal values and measurements collected with equipment $\mathrm{B}$ and $\mathrm{A}$ for the two experimen-

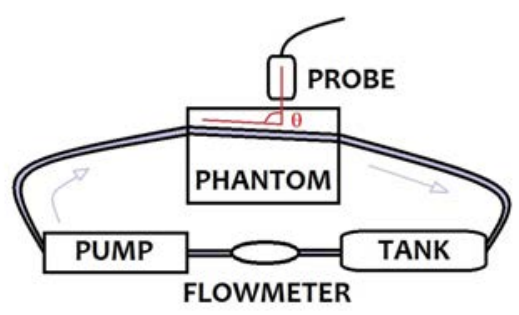

Figure 1. Scheme of the closed circuit composed by phantom, pump, tank and flowmeter. $\theta$ is the insonation angle.

Table 1. Parameter setting for equipment A.

\begin{tabular}{ll}
\hline Frequency of US beam & $5.6 \mathrm{MHz}$ \\
Maximum depth displayed & $72 \mathrm{~mm}$ \\
\hline Focus position & Set by the system \\
Gain & Set by the system \\
\hline Sample distance & $\begin{array}{l}\text { Central zone of the vessel to prevent the zone in which } \\
\text { the flow is not fully developed }\end{array}$ \\
Pulse repetition frequency (PRF) & $\begin{array}{l}\text { Between } 3 \mathrm{kHz} \text { and } 10 \mathrm{kHz} \text { with increasing flow, in order } \\
\text { to avoid the aliasing without using excessively high values }\end{array}$ \\
\hline
\end{tabular}


tal setups are reported in Table 5. For the ttest the threshold of $\mathrm{p}$-value for statistical significance is chosen equal to 0.05 . The equations of the linear regressions applied to the curves of mean values are reported in Figure 2. Slope and intercept of each curve are reported in Table 6.

\section{Discussion}

The main result of this study is that the equipment $\mathrm{B}$ has a better performance than equipment $\mathrm{A}$ in estimating velocity, as shown in Table 5 and 6 and in Figure 2. As confirmation of this, it is worth noting that the equipment $\mathrm{B}$ registered values very closed to those measured with the flowmeter. Nonetheless, considering the results shown in Table 4, the equipment $\mathrm{A}$ has a better repeatability and reproducibility than the equipment B. In other words, the equipment B shows higher accuracy but lower precision. This apparently contradictory result is probably due to the fact that the equipment A displays the TAV value calculated in a certain time frame, while the equipment $\mathrm{B}$ shows a single velocity measurement that is continually refreshed.

We think that the user interface of equipment $\mathrm{B}$ has to be improved showing also the TAV value, in order to consider the system for the clinical environment.

In conclusion, the equipment B seems to produce more accurate results than the equipment $\mathrm{A}$. This is an interesting outcome for all the fields where the velocity measurements play a critical role, such as diagnosis of carotid plaques and estimation of volume blood flow. For instance, our group has developed some lumped models based on volume blood flow estimation ${ }^{2,17,18}$ and one of the major limitation of such models was the low level of accuracy in flow quantification. For these reasons, we believe that more accurate velocity measurements can give a great improvement to both diagnostic responsibility and open scientific disputes. The use of equipment $B$ could be useful for the investigation of cerebral venous return both in physiological and in pathological condition, in particular avoiding as much as possible the errors linked to the blood flow quantification. Further studies will be required to analyse these issues in details.

\section{Conclusions}

In the present work it is shown that the new type of US device could be very attractive in future clinical environment, regarding diagnosis of cardiovascular diseases.

This system has the ability to measure the velocity with higher accuracy compared

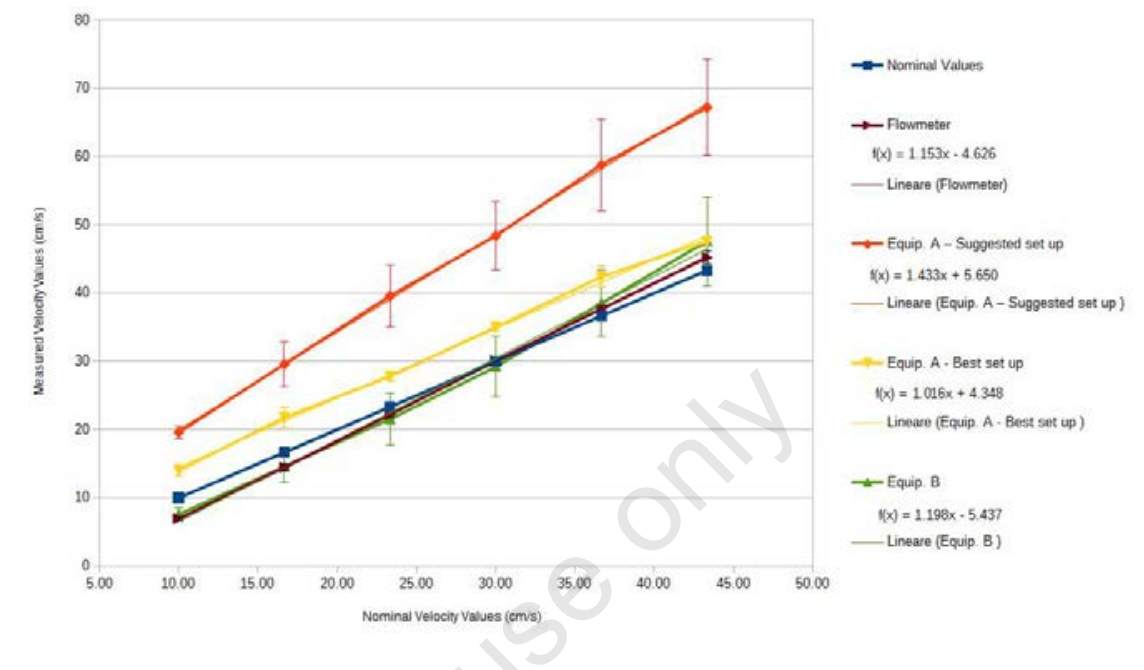

Figure 2. For each nominal velocity value, mean values of measurements collected with equipment $A$ and $B$, together with nominal values and flowmeter measurements are plotted. Respective SD are marked over each mean value. Linear regressions are drawn over each curve: their equations are reported in legend.

Table 2. Parameter setting for equipment $B$.

\begin{tabular}{ll}
\hline Frequency of US beam & $6.25 \mathrm{MHz}$ \\
Maximum depth displayed & $35 \mathrm{~mm}$ \\
\hline Focus position & Set in the same position of the marker \\
Time Gain Compensation (TGC) A & $30 \mathrm{~dB}$ \\
\hline TGC B & $0 \mathrm{~dB} / \mathrm{cm}$ \\
Sample distance & $\begin{array}{l}\text { Central zone of the vessel to prevent the zone in which } \\
\text { the flow is not fully developed }\end{array}$ \\
\hline PRF & $11,996 \mathrm{~Hz}$ \\
Speed of sound & $1480 \mathrm{~m} / \mathrm{s}$
\end{tabular}

Table 3. Tests done for the comparison between devices.

Verification of repeatability of measurements collected with equipment A during the same test

Verification of reproducibility of measurements collected with equipment A, using the same experimental setup in different sessions

Verification of repeatability of measurements collected with equipment $B$ during the same test

Verification of reproducibility of measurements collected with equipment $B$, using the same experimental setup in different sessions

Paired t-test between the set of measurements collected with the equipment A (Suggested setup), and the set of nominal values

Paired t-test between the set of measurements collected with the equipment A (Best setup) and the set of nominal values

Paired t-test between the set of measurements collected with the equipment $B$ and the set of nominal values

Paired t-test between the set of measurements collected with the equipment A (Suggested setup) and the set registered with the equipment B

Paired t-test between the set of measurements collected with the equipment A (Best setup) and the set collected with the equipment B

Calculation of intercept, slope and $r 2$ for each curve obtained with both equipments 
Table 4. Repeatability and reproducibility of measurements, tested on both equipments. Test Range of coefficient of variation (\%)

Repeatability equipment A $1-2$

Reproducibility equipment A (suggested setup) $7-12$

Reproducibility equipment A (best setup) $0-5$

Repeatability equipment $B$ $7-15$

Reproducibility equipment $B$ $13-17$

Table 5. Results of comparison between equipment A, B and nominal values.

\begin{tabular}{lcc}
\hline Paired t-test & Results of comparison & P-value \\
Equipment A (suggested setup) - Nominal values & Significantly different & $<1 \times 10^{-3}$ \\
Equipment A (suggested setup) - Equipment B & Significantly different & $<1 \times 10^{-4}$ \\
\hline Equipment A (best setup) - Nominal values & Significantly different & $<1 \times 10^{-5}$ \\
Equipment A (best setup) - Equipment B & Significantly different & $<1 \times 10^{-2}$ \\
\hline Equipment B - Nominal values & Not significantly different & $>0.8$ \\
\hline
\end{tabular}

Table 6. Slope and intercept of flowmeter, equipment A and equipment B.

\begin{tabular}{lccc} 
& Slope & Intercept & $\mathbb{R}^{2}$ \\
Flowmeter & 1.153 & 4.626 & 1.000 \\
Equipment A (suggested) & 1.433 & 5.650 & 0.999 \\
\hline Equipment A (best) & 1.016 & 4.348 & 0.998 \\
Equipment B & 1.198 & 5.437 & 0.995 \\
\hline
\end{tabular}

to the standard devices.

Further improvements could enhance the precision of Doppler measurements.

Moreover, future developments should take into account the B-mode, in order to complete the US quality control test on the device.

\section{References}

1. Gill RW. Measurement of blood flow by ultrasound: accuracy and sources of error. Ultrasound Med Bio 1985;11: 625-41.

2. Zamboni P, Sisini F, Menegatti E, et al. An ultrasonographic model to calculate the brain blood outflow through collateral vessels: a pilot study. BMC Neurology 2013;13-81.

3. Hoskins PR, Fish PJ, McDicken WN, Moran C. Developments in cardiovascular ultrasound: Part 2. Arterial applications. Med Biol Eng Comput 1998;36:259-69.

4. Sisini F, Tessari M, Gadda G, et al. An ultrasonographic technique to assess the jugular venous pulse: a proof concept. Ultrasound Med Bio 2015;41:1334-41.

5. Nakamura K, Qian K, Ando T, et al. Cardiac variation of internal jugular vein for the evaluation of hemodynamics. Ultrasound Med Bio 2016;42:176470.

6. Sisini F, Tessari M, Menegatti E, et al. Clinical applicability of assessment of jugular flow over the individual cardiac cycle compared with current ultrasound methodology. Ultrasound Med Bio 2016;1-14.

7. Menegatti E, Genova V, Tessari M, et al. The reproducibility of colour Doppler in chronic cerebrospinal venous insufficiency associated with multiple sclerosis. Int Angiol 2010;29: 121-6.

8. Dolic K, Marr K, Valnarov V, et al. Sensitivity and specificity for screening of chronic cerebrospinal venous insufficiency using a multimodal non-invasive imaging approach in patients with multiple sclerosis. Funct Neurol 2011;26:205-14
9. Ciccone MM, Galeandro AI, Scicchitano P, et al. Multigate quality Doppler profiles and morphological/hemodynamic alterations in multiple sclerosis patients. Curr Neurovasc Res 2012;9:120-7.

10. Monti L, Menci E, Piu P, et al. A sonographic quantitative cutoff value of cerebral venous outflow in neurologic diseases: a blinded study of 115 subjects. Am J Neuroradiol 2014 [Epub ahead of print].

11. Thibault P, Lewis W, Niblett S. Objective duplex ultrasound evaluation of the extracranial circulation in multiple sclerosis patients undergoing venoplasty of internal jugular vein stenoses: a pilot study. Phlebology 2015;30:98104.

12. Zamboni P. Why current Doppler ultrasound methodology is inaccurate in assessing cerebral venous return: the alternative of the ultrasonic jugular venous pulse. Behav Neurol 2016;2016:1-7.

13. Evans DH, McDicken WN, Skidmore R, Woodcock JP. Doppler ultrasound: physics, instrumentation, and clinical applications. New York, NY: John Wiley \& Sons; 1989.

14. Tortoli P, Bassi L, Boni E, et al. ULAOP: an advanced open platform for ultrasound research. IEEE Trans Ultrason Ferroelectr Freq Control 2009;56:2207-16.

15. Tortoli P, Dallai A, Boni E, et al. An automatic angle tracking procedure for feasible vector Doppler blood velocity measurements. Ultrasound Med Bio 2010;36;488-96.

16. Gerhard-Herman M, Gardin JM, Jaff M, et al. Guidelines for noninvasive vascular laboratory testing: a report from the American society of echocardiography and society of vascular medicine and biology. J Am Soc Echocardiog 2006;19:955-72.

17. Gadda G, Taibi A, Sisini F, et al. A new hemodynamic model for the study of cerebral venous outflow. Am J Physiol 2015;308:217-31.

18. Gadda G, Taibi A, Sisini F, et al. Validation of a hemodynamic model for the study of the cerebral venous outflow system using MR imaging and echocolor Doppler data. Am J Neuroradiol 2016;37:2100-9. 